\title{
Présentation de l'aménagement hydroélectrique du Pouget et du barrage de Pareloup (Aveyron)
}

\section{Description of Le Pouget hydroelectric installation and the Pareloup dam (Department Aveyron)}

\author{
EDF Production Transport - Energie Midi-Pyrénées ${ }^{(1)}$ \\ (1) 77, chemin des courses F-31057 Toulouse cedex.
}

Résumé. - Description des différents barrages et usines constituant l'aménagement hydroélectrique du Pouget dans l'Aveyron et mode de fonctionnement.

Mots-clés. - Hydroélectricité, barrage, retenue artificielle.

Abstract. - Description of the various dams and power stations constituting the Le Pouget hydroelectric installation, with their modes of operation.

Key words. - Hydroelectric power station, dam, artificial reservoir

\section{AMÉNAGEMENT DU POUGET}

L'aménagement du Pouget se situe au centre du département de l'Aveyron (fig. 1). Il utilise le dénivelé existant entre le plateau du Levezou, plateau granitique faiblement vallonné dont l'altitude varie de $700 \mathrm{~m}$ à $900 \mathrm{~m}$, et le sillon creusé par le Tarn $500 \mathrm{~m}$ plus bas.
Cet aménagement est organisé autour du lac de Pareloup, réserve de $169 \mathrm{hm}^{3}$ qui occupe une dépression particulièrement favorable sur le cours du Vioulou, affluent rive gauche du Viaur, à $30 \mathrm{~km}$ au Sud-Est de Rodez.

Sa très grande capacité, au $16^{e}$ rang des réserves de France permet d'emmagasiner l'eau pendant les saisons de moindre besoin en électricité pour l'utiliser pendant les saisons 


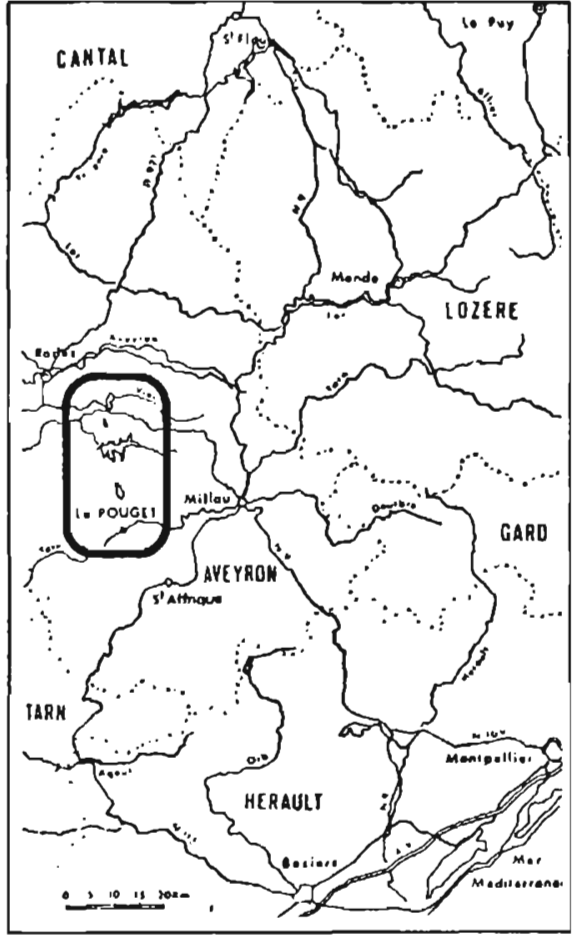

Fig. 1. - Situation géographique de l'aménagement hydroélectrique du Pouget.

Fig. 1. - General overview of Le Pouget hydroelectric installation.

de fortes demandes (principalement I'hiver). Aussi, outre les apports du Vioulou, la réserve de Pareloup stocke les apports du Viaur, par l'intermédiaire des retenues de Pont de Salars $\left(20 \mathrm{hm}^{3}\right)$ et Bage $\left(4 \mathrm{hm}^{3}\right)$ qui fonctionnent en vases communicants. La station de pompage de Bage $\left(14 \mathrm{~m}^{3} / \mathrm{s}\right)$ remonte ces apports sur les $98 \mathrm{~m}$ de dénivellation séparant Bage de Pareloup, la jonction se faisant par une galerie de $6,4 \mathrm{~km}$ de long.

La partie aval de l'aménagement assure la jonction avec la centrale du
Pouget (fig. 4) qui restitue au Tarn l'eau emmagasinée dans le lac de $\mathrm{Pa}$ reloup. Elle est constituée essentiellement du lac de Villefranche-de-Panat $\left(11 \mathrm{hm}^{3}\right)$, réserve intermédiaire drainant l'extrémité Sud du plateau.

La centrale d'Alrance permet d'exploiter la dépression de $80 \mathrm{~m}$ existant entre Pareloup et Villefranche-de-Panat. Elle est située au débouché aval de la galerie de $10,9 \mathrm{~km}$ issue de $\mathrm{Pa}$ reloup.

Enfin 2 galeries de $5,3 \mathrm{~km}$ et 2 conduites forcées de $1,2 \mathrm{~km}$ chacune, joignent Villefranche de Panat à la centrale du Pouget, exploitant par là une chute de $461 \mathrm{~m}$. A noter le petit lac de Saint-Amans $\left(0,8 \mathrm{hm}^{3}\right)$, en tête des conduites forcées, qui joue le rôle d'une cheminée d'équilibre.

L'aménagement du Pouget a été récemment augmenté d'une station de pompage $\left(6,6 \mathrm{~m}^{3} / \mathrm{s}\right)$ au Truel permettant de remonter les eaux du Tarn dans la retenue de Villefranche-dePanat par l'une des conduites forcées et galeries décrites plus haut.

Cette adjonction prendrait toute sa valeur avec le projet de la pompe d'Alrance qui prolongerait la réalisation précédente en remontant les eaux du lac de Villefranche-de-Panat dans la retenue saisonnière de Pareloup.

L'ensemble de ce dispositif assez complexe est représenté en masse sur la figure 2 et au travers d'un schéma hydraulique sur la figure 3 . 
Retenue de

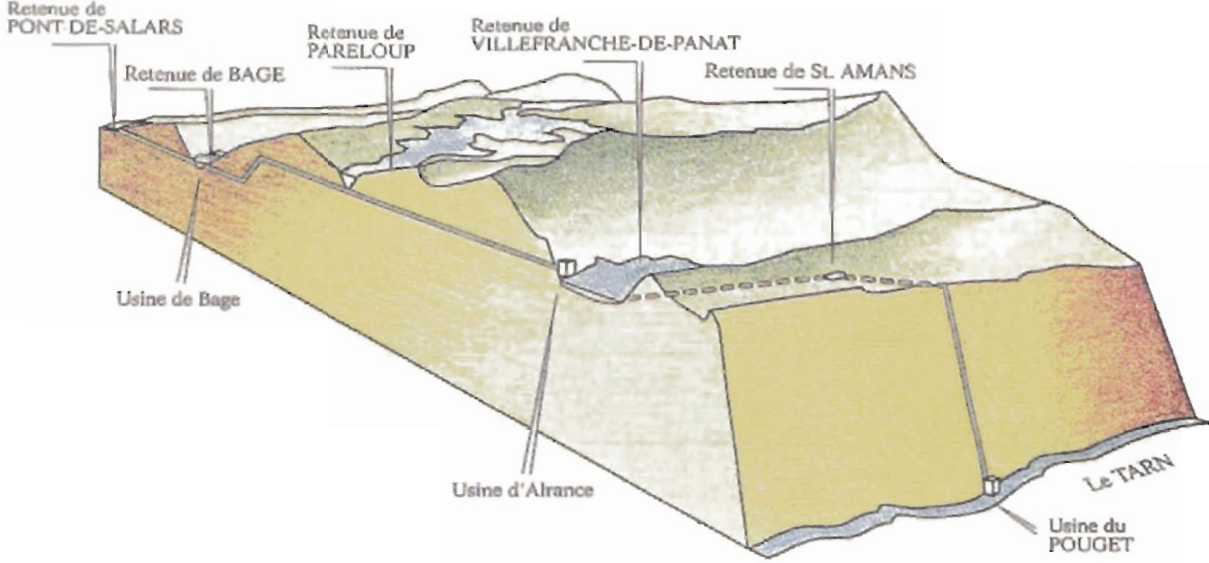

Fig. 2. - Maquette des installations.

Fig. 2. - Model of installations.

\section{$\rightarrow$ L'AMÉNAGEMENT DU POUGET EN CHIFFRES}

\section{Barrage de PONT-DE-SALARS}

Voüte mince en béton de $35,5 \mathrm{~m}$ de hauteur Superficie : 200 ha, Volume total : $20 \mathrm{hm}^{3}$ Volume utile : $14,55 \mathrm{hm}^{3}$

\section{Barrage de BAGE}

Voûte mince en béton de $27,45 \mathrm{~m}$ de hauteur Volume iotal: $4 \mathrm{hm}^{3}$, Volume utile: $3,09 \mathrm{hm}^{3}$ Superficie : 53 ha

\section{Usine de BAGE}

Débit max. pompable: $14 \mathrm{~m}^{3} / \mathrm{s}$

Hauteur de chute brute : $98 \mathrm{~m}$

Puissance max.: 16,2 MW,

\section{Barrage de PARELOUP}

Voir Paragraphe 2

\section{Usine d'ALRANCE}

Débit max., turbinable $24 \mathrm{~m}^{3} / \mathrm{s}$ Hauteur de chute brute: $79,8 \mathrm{~m}$

Puissance max. : 11,15 MW

Production moyenne annuelle:

$30.16^{6} \mathrm{kWh}$

\section{Barrage de VILLEFRANCHE-DE-PANAT}

Type poids en béton de $17,10 \mathrm{~m}$ de hauteur

Superficie: 197 ha, Volume total:

$11 \mathrm{hm}^{3}$

Volume utile : $8,66 \mathrm{hm}^{3}$

Barrage de SAINT-AMANS

Type poids en béton de $26,5 \mathrm{~m}$ de hauteur

Volume total : $0,8 \mathrm{hm}^{3}$. Volume utile:

$0,65 \mathrm{hm}^{3}$

\section{Usine de POUGET}

Débit max. turbinable: $105,6 \mathrm{~m}^{3} / \mathrm{s}$

Hauteur de chute brute: $461,2 \mathrm{~m}$

Puissance max. : $440 \mathrm{~mW}$

Production moyenne annuelle:

$297.10^{6} \mathrm{kWh}$ 


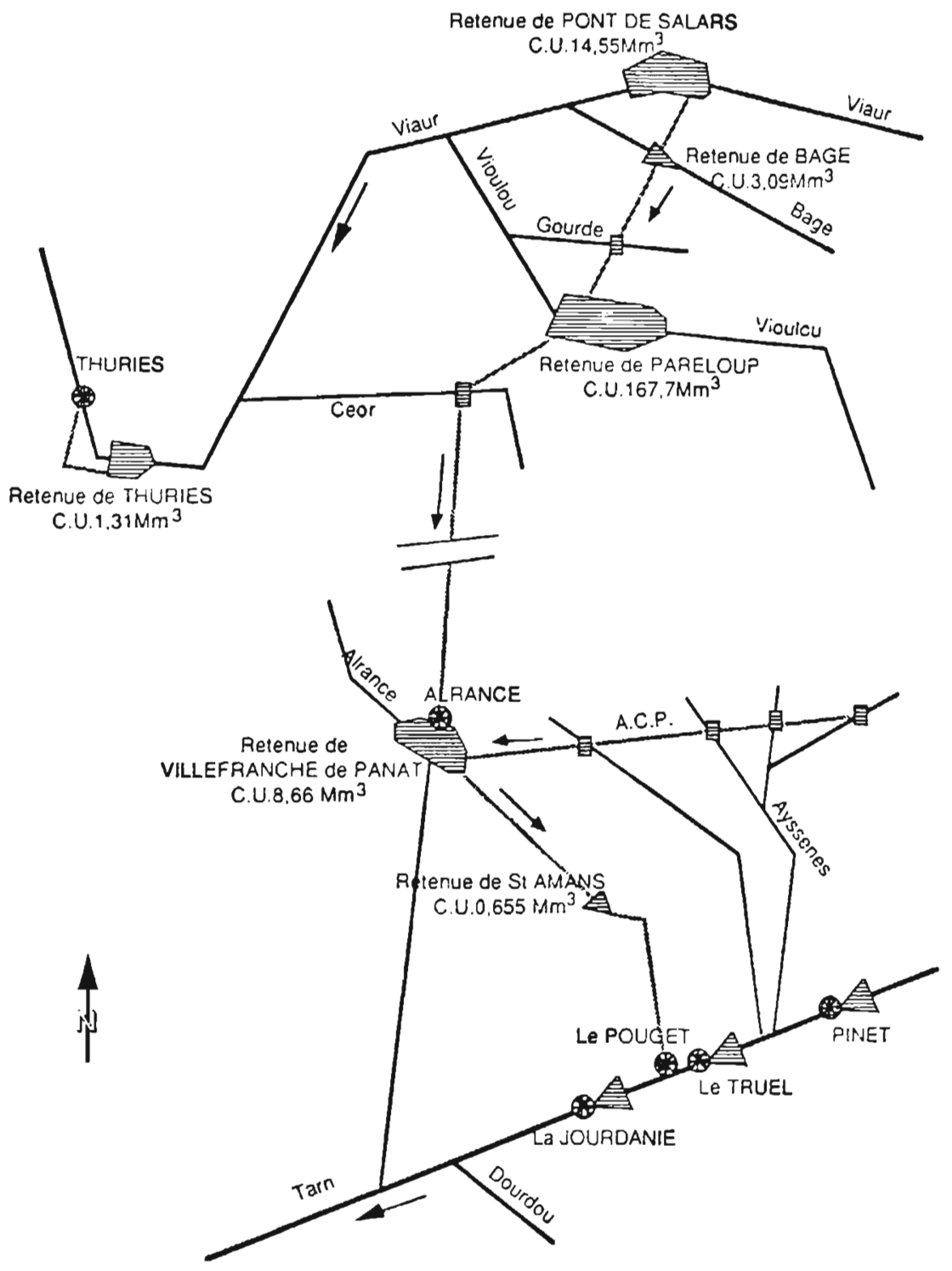

Fig. 3. - Schéma hydraulique des bassins Tarn-Viaur.

Fig. 3. - Hydrological diagram of Tarn and Viaur basins. 


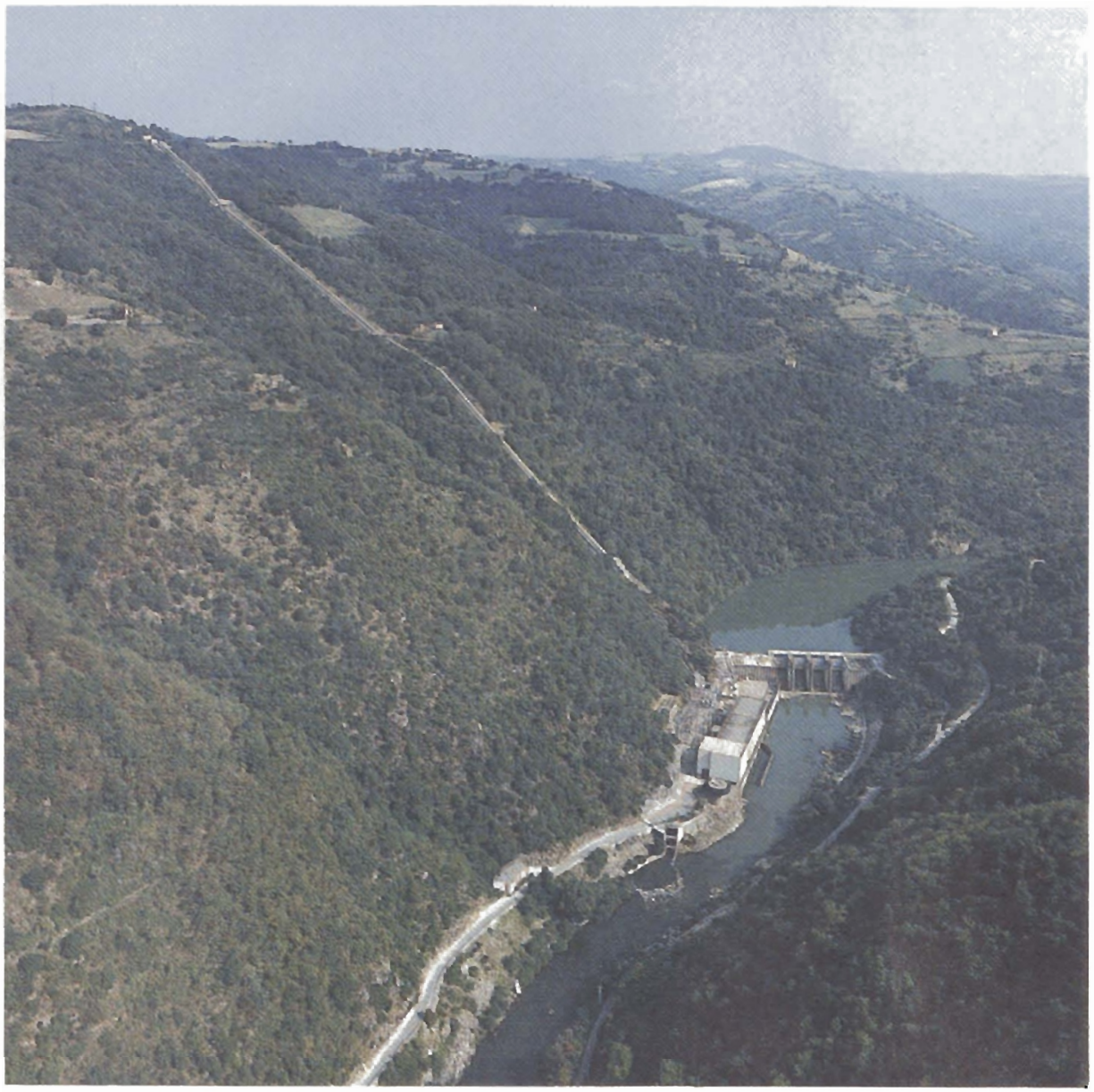

Fig. 4. - Vue de l'usine hydroélectrique du Pouget.

Fig. 4. - Le Pouget hydroelectric power station.

\section{BARRAGE DE PARELOUP}

Le barrage de Pareloup, sur le Vioulou est une voûte mince en béton de $43,45 \mathrm{~m}$ de hauteur réalisée de 1949 à 1951. Ii est situé à $30 \mathrm{~km}$ au Sud-Est de Rodez.

\section{Les Apports}

La retenue de Pareloup est alimentée par le Vioulou, dans la vallée duquel le barrage est construit. Par l'intermédiaire de la station de pompage de Bage, cette retenue est éga- 
lement alimentée par le Viaur et le Bage dont les apports sont stockés dans les retenues de Pont-de-Salars et Bage.

La retenue de Pareloup dispose donc des apports :

- du bassin versant de $160 \mathrm{~km}^{2}$ sur le Vioulou complété par les bassins versants de petites adductions, essentiellement la Gourde (BV de $4 \mathrm{~km}^{2}$, apport moyen de $77,4 \mathrm{l} / \mathrm{s}$ ) et le Ceor (BV de $4 \mathrm{~km}^{2}$, apport moyen de $77,4 \mathrm{l} / \mathrm{s})$. Le débit moyen mensuel (Gourde + Céor + Vioulou + BV gravitaire de Pareloup) correspond à $3,25 \mathrm{~m}^{3} / \mathrm{s}$;

- du bassin versant de $216 \mathrm{~km}^{2}$ sur le Viaur et le Bage. Le débit moyen mensuel apporté par le pompage de Bage est de $4,21 \mathrm{~m}^{3} / \mathrm{s}$. La galerie amène les eaux dans la retenue de Pareloup à la cote 768 NGF.

Toutefois il faut garder à l'esprit qu'il y a de grandes variations possibles des apports totaux dans la retenue de Pareloup.

\section{- variation d'une année sur l'autre:}

De 1961 à 1988, durant les mois de décembre, on constate, en débit moyen mensuel, un minimum de $0,46 \mathrm{~m}^{3} / \mathrm{s}$ et un maximum de $37,2 \mathrm{~m}^{3} / \mathrm{s}$ pour une moyenne de $12,1 \mathrm{~m}^{3} / \mathrm{s}$, soit un écart de 1 à 80 . Toujours de 1961 à 1988 et en débit moyen mensuel, on constate durant les mois d'août, un minimum de $0,15 \mathrm{~m}^{3} / \mathrm{s}$ et un maximum de $5,50 \mathrm{~m}^{3} / \mathrm{s}$ pour une moyenne de $1,0 \mathrm{~m}^{3} / \mathrm{s}$, soit un rapport de 1 à 36 .

- mais aussi variation dans l'année:
Entre été et hiver où le débit moyen mensuel interannuel sur la période 1961 à 1988 passe de $1,0 \mathrm{~m}^{3} / \mathrm{s}$ l'été à $14 \mathrm{~m}^{3} / \mathrm{s}$ l'hiver, avec des pointes comme l'année 1961 (juillet/août: $0,15 \mathrm{~m}^{3} / \mathrm{s}$, janvier/février: $19,6 \mathrm{~m}^{3} / \mathrm{s}$, soit un rapport de 1 à 130).

- et également au cours d'un même mois: en mars 1988 on constate en débits moyens journaliers : $5,18 \mathrm{~m}^{3} / \mathrm{s}$ le 12 et $57,0 \mathrm{~m}^{3} / \mathrm{s}$ le 18 pour une moyenne mensuelle de $18,3 \mathrm{~m}^{3} / \mathrm{s}$, soit un rapport de 1 à 11 à quelques jours d'intervalle.

\section{Les sorties d'eau}

Les sorties d'eau sont de deux sortes: le débit turbiné à l'usine d'Alrance, prélevé dans la retenue à la cote 768 NGF et le débit réservé du Vioulou, de 103 l/s, soutiré sur les vannes de fond à la cote 765 NGF.

L'article 50 du cahier des charges de la concession du Pouget prévoit la possibilité d'un prélèvement d'un débit de $15 \mathrm{l} / \mathrm{s}$ pour l'alimentation en eau potable de la ville de Rodez et de $73 \mathrm{l} / \mathrm{s}$ pour le Syndicat des Eaux du Segala. Par conventions, la ville de Rodez est autorisée à prélever jusqu'à $50 \mathrm{l} / \mathrm{s}$ et le Syndicat du Segala jusqu'à $127 \mathrm{l} / \mathrm{s}$.

Ceci représente en moyenne, pour Rodez environ $1 \mathrm{hm}^{3}$ par an et pour le Segala de 2,2 à $2,5 \mathrm{hm}^{3}$ par an. 


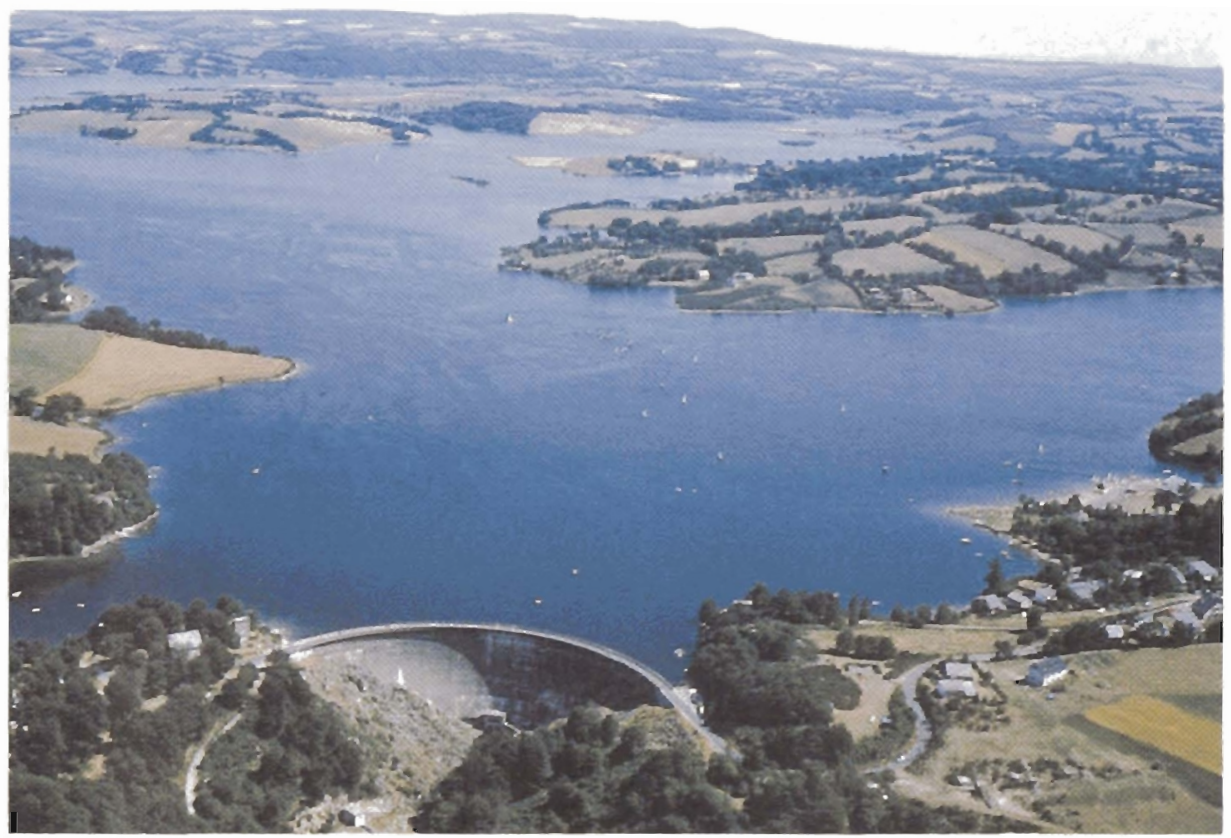

Fig. 5. - Vue générale de ta retenue de Pareloup.

Fig. 5. - General overview of Pareloup lake.

\section{La retenue}

Côte de retenue normale:

Côte minimale d'exploitation:

805,00 NGF

$775,00 \mathrm{NGF}$

Côte minimale turbinable :

$771,60 \mathrm{NGF}$

Volume de la retenue:

169 Millions de $\mathrm{m}^{3}$

Volume utile de la retenue:

167,7 Millions de $\mathrm{m}^{3}$

Surface de la retenue à la côte 805,00 NGF :

1260 ha.

La retenue de Pareloup est la $5^{\ominus}$ retenue hydroélectrique de France par sa superficie et la $14^{\circ}$ par son volume (fig. 5).

\section{Le barrage}

Hauteur maxi, au-dessus du terrain naturel: $43,45 \mathrm{~m}$

Hauteur maxi, au-dessus des fondations: $47,45 \mathrm{~m}$

Altitude du couronnement: $806,45 \mathrm{~m}$ NGF

Largeur maxi. du barrage au niveau des fondations: $15,87 \mathrm{~m}$

Largeur du barrage au couronnement : $2,77 \mathrm{~m}$

Longueur au couronnement: $232 \mathrm{~m}$

Volume du barrage: $35000 \mathrm{~m}^{3}$

Le barrage de Pareloup est aujourd'hui le $16^{\circ}$ barrage de France par sa hauteur. 